Revue d'histoire de l'Amérique française

REVUE D.HISTOIRE DE L'AMÉRIQUE FRANÇAISE

\title{
La Louisiane et les colonies espagnoles d'Amérique (1731-1748)
}

\section{Jean-Marie Loncol}

Volume 18, numéro 2, septembre 1964

URI : https://id.erudit.org/iderudit/302384ar

DOI : https://doi.org/10.7202/302384ar

Aller au sommaire du numéro

Éditeur(s)

Institut d'histoire de l'Amérique française

ISSN

0035-2357 (imprimé)

1492-1383 (numérique)

Découvrir la revue

Citer ce document

Loncol, J.-M. (1964). La Louisiane et les colonies espagnoles d'Amérique (1731-1748). Revue d'histoire de l'Amérique française, 18(2), 313-315.

https://doi.org/10.7202/302384ar d'utilisation que vous pouvez consulter en ligne.

https://apropos.erudit.org/fr/usagers/politique-dutilisation/ 


\title{
BIBLIOGRAPHIE
}

\section{LA LOUISIANE ET LES COLONIES ESPAGNOLES D'AMÉRIQUE (1731-1748)*}

\author{
A - MANUSCRITS
}

Nous avons consulté d'après les copies de la Bibliothèque du Congrès, à Washington.

a. Archives des Colonies [AC]

Série B. - Contient la correspondance du ministère de la Marine adressée aux administrateurs des colonies françaises d'Amérique. Nous avons puisé dans les volumes $55,57,59,61,64$, 65, 66, 83, 85, couvrant les années 1731 à 1747 .

Série C13A. - Contient la correspondance des administrateurs de la Louisiane adressée au ministère de la Marine. Nous avons puisé dans les volumes $19,20,22,30,32,43$, s'échelonnant des années 1734 à 1761.

Collection F3 - Collection Moreau de Saint-Méry. Parmi les documents se rapportant à l'histoire de la Louisiane, nous avons relevé, au vol. 24 (fol. 247-248), la "Copie de l’opposition du Seigneur Gonzalès enseigne de la Compagnie des Adays et Lieutenant general de Mr de Sandoval capitaine d'Infanterie Espagnole et gouverneur des provinces de la Nouvelle-Philippine à M. de St Denis commandant des Natchitoches", ainsi que la "Réponse à cette opposition".

b. Affaires Etrangères [AE]

Mémoires et Documents, Espagne. - Au vol. 525 (fol. 137), la réponse de Don José Patino à un office de l'ambassadeur de France, au sujet de l'accueil réservé aux vaisseaux français à Cuba (19 août 1735).

* Voir l'article de M. Loncol, sur ce sujet, aux pages 196-201 de cette même livraison. 


\section{B - GuIDES ET INVENTAIRES}

Cuthbert, Norma B., American Manuscript Collections in the Huntington Library for the History of the 17th and 18th Centuries. Huntington Library Lists, No. 5. San Marino, Calif., 1941.

Description des documents Vaudreuil, Collection Loudoun. [H M LO]; nous n'y avons cependant pas trouvé un inventaire détaillé des documents qui nous intéressaient.

Hill, Roscoe R., éd., The National Archives of Latin America. Joint Committee on Latin American Studies, Miscellaneous Publication No 3. Cambridge, Mass., 1945.

Ce guide semble commode pour une étude de notre sujet du point de vue espagnol. Signalons, en ce qui concerne les archives cubaines, les collections suivantes: Correspondencia general, 1716-1887; Floridas, 1737-1823 ; Gobierno Superior Civil, 1700-1880; Papeles de asuntos politicos, 1721-1898; Intendencia de Hacienda, 1712-1862; Libros varios de las administraciones espanoles. Aux archives mexicaines: Archivo general de guerra (Epoca colonial) ; Correspondencia de los Virreyes; General de Parte; Historia, Epoca colonial, 1697-1821; Marina; Provincias internas; Reales Cédulas.

Surrey, N. M., éd., Calendar of Manuscripts in Paris Archives and Libraries Relating to the History of the Mississipi Valley to 1830. 2 vol. Carnegie Institution of Washington Department of Historical Research, 1926-1928. Instrument de travail très utile, même si les références manquent parfois de précision. C'est à partir de cet index chronologique, qu'après avoir noté ce qui se rapportait aux relations Louisiane-colonies espagnoles, nous avons choisi les documents examinés dans ce travail.

\section{C - COLLECTIONS DE SOURCES CONTEMPORAINES}

Delanglez, Jean, éd., "Louisiana in 1717: Mémoire sur la Louisiane pour estre présenté, avec la Carte de ce Païs, au Conseil Souverain de Marine, par F. Le Maire, P.P. Miss. op." Revue d'histoire de l'A mérique française [RHAF], 3 (19491950) : $94-100 ; 256-269 ; 423-446$.

Rapport de l'Archiviste de la province de Québec [RAPQ] pour 1923-24. Québec, 1924.

"Réflexions sur la campagne prochaine - examen de cette question: si Québec pris le Canada l'est; de cette autre: si le 
Canada perdu, la France peut soutenir la Louisiane. Dispositions à faire relatives à cet objet." P. 16-18. Il s'agit d'un des mémoires emportés par Bougainville lors de sa mission en France en 1758-59.

Nous n'avons pas consulté les textes suivants:

Devron, G., éd., "A Chapter of Colonial History, Louisiana, 1717 to 1752." [Document anonyme.] Louisiana Historical Quarterly [LHQ], 6 (1923) : 543-567.

"Records of the Superior Council of Louisiana (1743-1753)." LHQ, 11 (1928) à 22 (1939). Les copies de ces registres se trouvent d'ailleurs à la bibliothèque de l'Université Tulane (Nouvelle-Orléans) ; le coût relativement élevé des photocopies nous a empêché de poursuivre les recherches sur ces documents.

Shelby, C. C., éd., "Grenier's Journal of His Voyage to Vera Cruz, 1745." LHQ, 21 (1938) : 631-655.

\section{D - MONOGRAPHIES}

Frégault, Guy, Le Grand Marquis; Pierre de Rigaud de Vaudreuil et la Louisiane. Montréal, 1952.

Etudie également la Louisiane avant 1743. Parmi les activités de Vaudreuil, les relations avec les colonies espagnoles figurent en bonne place; bien entendu, le rôle personnel du gouverneur est mis ici en évidence. Comme les questions diplomatiques restent malgré tout accessoires dans le cadre de cet ouvrage, l'auteur n'indique que quelques sources espagnoles.

Lamontagne, Roland, La Vie et l'Oeuvre de Pierre Bouguer (Paris, 1964).

Surrey, N. M., The Commerce of Louisiana During the French Regime, 1699-1763. New York, 1916. Cet ouvrage que signale M. Frégault n'a pas été consulté.

\section{E - PÉRIODIQUES}

La Revue d'histoire de l'Amérique française et la Canadian Historical Review, que nous avons compilées, n'offrent guère d'articles se rapportant à notre sujet; le Louisiana Historical Quarterly et la Mississipi Valley Historical Review n'ont pas été consultés.

JEAN-MARIE LONCOL 